\title{
Mixed convection stagnation-point flow over a vertical plate with prescribed heat flux embedded in a porous medium: Brinkman-Extended Darcy formulation.
}

\begin{abstract}
This article considers the problem of mixed convection stagnation-point flow towards a vertical plate embedded in a porous medium with prescribed surface heat flux. It is assumed that the free stream velocity and the surface heat flux vary linearly from the stagnation point. Using a similarity transformation, the governing system of partial differential equations is transformed into a system of ordinary differential equations, before being solved numerically by a finite-difference method. The features of the flow and the heat transfer characteristics are analyzed and discussed. It is found that dual solutions exist for both buoyancy assisting and opposing flows.
\end{abstract}

Keyword: Stagnation-point; Porous medium; Heat transfer; Mixed convection. 